\title{
Wastewater diffusive dilution and sedimentation of the fine contaminated particles for nonuniform flow in open channels
}

\author{
Anton Lyapin ${ }^{1}$ and Valery Lyapin ${ }^{2 *}$ \\ ${ }^{1}$ Moscow State University of Civil Engineering, Yaroslavskoye shosse, 26, Moscow, Russia, 129337 \\ ${ }^{2}$ Journal «Power Technology and Engineering», 2/1 Voroncovsky Pereulok, Moscow, Russia
}

\begin{abstract}
The influence of non-uniformity on mass transfer processes in open channels have been investigated under the action of urbanization factors. The study is related to the urgent problem of environmental degradation of water objects in urbanized areas. It is known that the water quality in the water objects depends on the manner in which the contaminants spread how they mix with the river water and diluted by it. The main results of the study consist of recommendations to incorporate non-uniformity factor to the calculation of diffusion dilution of wastewater and prediction of river processes. So the effect of the flow non-uniformity on the diffusion model of pollutants dilution and diffusion coefficient have been investigated. Formulas for the concentration profiles calculating and the average concentration of fine particulate matter in nonuniform gradually varied flow were presented. The deposition length of suspended contaminants were received, based on the hydraulic resistance laws of nonuniform gradually varied flow.
\end{abstract}

Keywords: nonuniform flow, diffusive dilution, hydraulic resistance, suspended contaminants

\section{Introduction}

The obvious result of intensive use of water objects in urban areas is the deterioration of water quality. Industrial and domestic wastewater from urbanized areas contain the large amount of different pollutants (oil, acid and alkali, suspended solids, salts, heavy metal ions, organics, solvents, surfactants, and etc.) in the form of soluble and suspended substances. Suspended solids are sandy, silty or clay particles of different sizes, which adsorb the surface contaminants. The subsequent deposition of suspended matter is one of the most important factors of soil pollution. In periods of most active riverbed processes is an active mixing of soils with pollutants of the riverbed. This leads to a more intense pollution of ground layers of substantial thickness (up to $1 \mathrm{~m}$ and more) [1]. Contaminated bed soils can be an independent source of water pollution for a significant period after termination of the wastewater discharge. The main sources of fine turbidity and organic contaminants are shown in Fig. 1.

\footnotetext{
* Corresponding author: 1yapinanton@gmail.com
} 

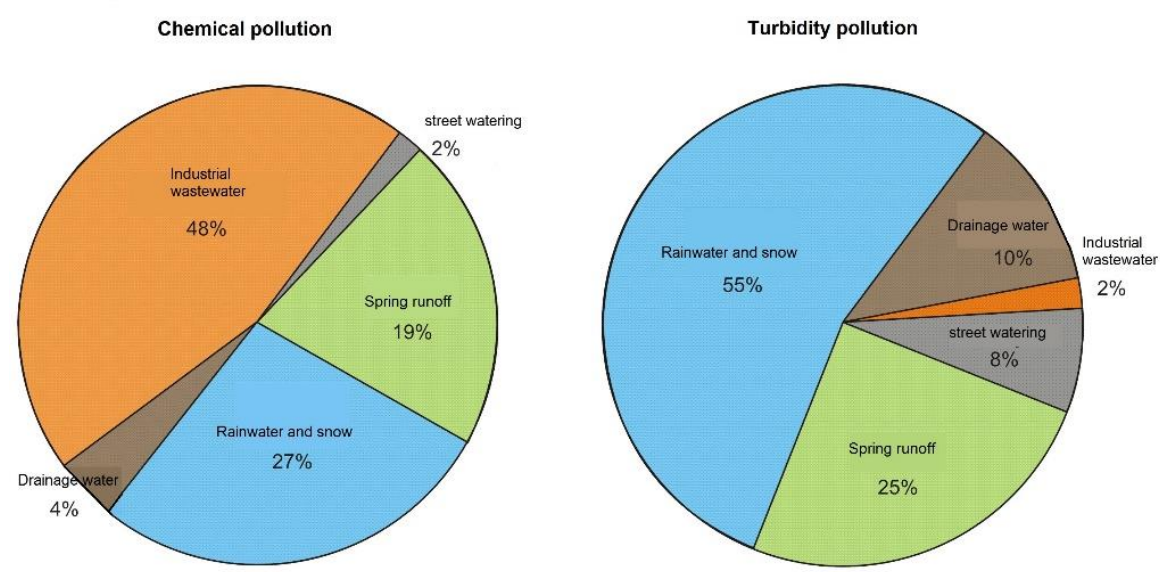

Fig. 1. Moscow river pollution sources according to MosvodokanalNIIproekt data

As can be seen from the figure the main source of turbidity pollution is runoff from urban areas. About half of all chemical contamination of organic nature constitute treated industrial and household wastewater. Long-term anthropogenic and technogenic impact on water flow leads to changes in water flow and sediments as well, as the channel geometry. The effect of these factors is reshaping and changing the river network, in some cases, negative and irreversible. Flow nonuniformity what is present in natural watercourses [2] plays important role in all above mentioned processes.

Dispersion of soluble pollutants in the aqueous medium refers to a category of nonequilibrium processes. These processes are due to varying concentrations of discharged pollutants in the river. Diffusion model of turbulent transport of conservative pollutants is described by Fick's law [3]

$$
j=-D_{T} \frac{\partial c}{\partial y}
$$

$\mathrm{j}$ - diffusion flux, of which the dimension is amount of substance per unit area per unit time; $D_{T}$ - diffusion coefficient; $\partial \mathrm{c} / \partial \mathrm{y}$ - gradient of concentration; $y$ - vertical coordinate.

\section{The diffusion coefficient for nonuniform flow}

The diffusion coefficient characterizes the transfer of substance by chaotic turbulent motion and depends on the hydrodynamic flow characteristics. Since the flow of water in the natural watercourses is generally nonuniform let's investigate the influence of the factor of nonuniformity on the value of the coefficient of turbulent diffusion assuming the same depth values and the average flow velocities in the considered cross-sections of nonuniform and "equivalent" uniform flow. Especially that currently practiced approach to the calculation of the hydrodynamic characteristics of the middle section of gradually varied nonuniform flow needs, as shown by recent studies [2,4], in serious correction. Consider this question based on the identity determining of the diffusion coefficient $D_{T}$ and the eddy viscosity $v_{T}[3]$.

Determine the coefficient of eddy viscosity in open nonuniform gradually varied flow using relationship [2]

$$
v_{T}=\kappa U_{*} h \eta(1-\eta)
$$

$\kappa$ - von Kármán parameter; $U_{*}$ - friction velocity; $h$ - depth of flow; $\eta=y / h$ dimensionless distance from the bottom.

In the case of an "equivalent" uniform flow, the Eq. (2) will differ by von Kármán parameter value $\left(\kappa_{p}\right)$ and friction velocity value $\left(U_{* \mathrm{p}}\right)$ 


$$
v_{T \mathrm{p}}=\kappa_{p} U_{* p} h \eta(1-\eta)
$$

After dividing Eq. (2) by Eq. (3) and elementary mathematical transformations we have

$$
\frac{v_{T}}{v_{T \mathrm{p}}}=\frac{\kappa}{\kappa_{p}} \frac{U_{*}}{U_{* p}} \text {. }
$$

Thus, the influence of nonuniformity on the eddy viscosity (diffusion coefficient) can be identify analyzing the changes of parameters $\kappa$ and $U_{*}$ in a nonuniform flow.

Following F.Engelund [6], we can write the one-dimensional energy and momentum equations for nonuniform flow:

$$
\begin{gathered}
\frac{d}{d x}\left(h+\frac{\alpha q^{2}}{2 g h^{2}}\right)=i-\frac{1}{2} \lambda \frac{q^{2}}{g h^{3}}, \\
\frac{d h}{d x}+\frac{1}{h} \frac{d}{d x}\left(\frac{\beta q^{2}}{g h}\right)=i-\frac{1}{2} C_{f} \frac{q^{2}}{g h^{3}},
\end{gathered}
$$

$\lambda$ - hydraulic resistance coefficient of nonuniform flow defined by the hydraulic radius; $a, \beta$ - kinetic energy and momentum correction factors; $q$ - specific discharge; $i$ - bottom slope; $C_{f}=2\left(U_{*} / V\right) 2$ - skin friction coefficient ( $V$ - mean velocity); $x$ - longitudinal coordinate; $g$ acceleration due to gravity.

Subtracting Eq. (5) from Eq. (6), we will find after simple transformations

there $m=3,12 \lambda[6]$.

$$
\lambda=C_{f}+4 m \frac{d h}{d x}
$$

Eq. (7) may be rewritten as

$$
C_{f}=\lambda\left(1-12,5 \frac{d h}{d x}\right) .
$$

For decelerated flows $(d h / d x>0)$ in a range $d h / d x \leq 0,02$ is valid relationship [4, 5]

$$
\lambda=\lambda_{p}\left(1+21,2 \frac{d h}{d x}\right)
$$

$\lambda_{p}$ - hydraulic resistance coefficient of "equivalent" uniform flow.

Substituting Eq. (9) into Eq. (8), we obtain

$$
C_{f}=\lambda_{p}\left(1-12,5 \frac{d h}{d x}\right)\left(1+21,2 \frac{d h}{d x}\right)
$$

Considering that $C_{f}=2\left(U_{*} / V\right)^{2}$ and $\lambda_{p}=2\left(U_{*} p / V\right)^{2}$ we can find

$$
\frac{U_{*}}{U_{* p}}=\left(1-12,5 \frac{d h}{d x}\right)\left(1+21,2 \frac{d h}{d x}\right) .
$$

Opening brackets and neglecting small terms we have

$$
\frac{U_{*}}{U_{* p}} \approx 1+8,7\left|\frac{d h}{d x}\right| \text {. }
$$

For accelerated flows $(d h / d x<0) \lambda=\lambda_{p}[4,5]$ and a similar dependence has the form

$$
\frac{U_{*}}{U_{* p}}=\left(1-12,5 \frac{d h}{d x}\right) \text {, or } \frac{U_{*}}{U_{* p}}=\left(1+12,5\left|\frac{d h}{d x}\right|\right) \text {. }
$$

Processing of experimental data I. Nikuradze [7], F.Donhe [8] and A.G.Gurzhienko [9] for flat decelerated flows led [4] to the next Eq. for the von Kármán parameter

$$
\frac{\mathrm{\kappa}}{\mathrm{\kappa}_{p}}=1+76,5 \frac{d h}{d x} \text {. }
$$
flow

After substituting Eq. (12) and Eq. (14) into Eq. (4) it is possible to find for decelerated

$$
\frac{\kappa}{\kappa_{p}} \frac{U_{*}}{U_{* p}}=\frac{v_{T}}{v_{T \mathrm{p}}}=\left(1+76,5 \frac{d h}{d x}\right)\left(1+8,7 \frac{d h}{d x}\right), \text { or } \frac{v_{T}}{v_{T \mathrm{p}}}=1+85.2\left|\frac{d h}{d x}\right| .
$$

A similar dependence for accelerated flow can be obtained after the substitution Eq. (13) into Eq. (4)

$$
\frac{\kappa}{\kappa_{p}} \frac{U_{*}}{U_{* p}}=\frac{v_{T}}{v_{T \mathrm{p}}}=\frac{\kappa}{\kappa_{p}}\left(1+12,5\left|\frac{d h}{d x}\right|\right) .
$$


In accordance with the well-known power-law dependence for velocity profile [10] the equality of the hydraulic resistance coefficients for accelerated and "equivalent" uniform flow simultaneously assumes the identity of the distributions of mean velocity in the comparable flows. Taking on this basis $\kappa=\kappa_{p}$ we find

$$
\frac{\kappa}{\kappa_{p}} \frac{U_{*}}{U_{* p}}=\frac{v_{T}}{v_{T \mathrm{p}}}=1+12,5\left|\frac{d h}{d x}\right| .
$$

Based on the identity of determination the coefficient $D_{T}$ and the eddy viscosity $v_{T}$ [3] we can turn from Eq. (15) and Eq. (17) to the analogous dependences for the diffusion coefficients $D_{T}$.

For decelerated flow

For accelerated flow

$$
\frac{D_{T}}{D_{T \mathrm{p}}}=1+85.2\left|\frac{d h}{d x}\right|
$$

$$
\frac{D_{\mathrm{T}}}{D_{\mathrm{Tp}}}=1+12,5\left|\frac{d h}{d x}\right| .
$$

Comparing the values of diffusion coefficient for decelerated (DT3) and accelerated (DTy) flows in accordance with the Eq. (18) and Eq. (19) we obtain

$$
\frac{D_{\mathrm{T} 3}}{D_{\mathrm{Ty}}}=\frac{1+85.2\left|\frac{d h}{d x}\right|}{1+12,5\left|\frac{d h}{d x}\right|} \approx 6,8-\frac{5,8}{1+12,5\left|\frac{d h}{d x}\right|} .
$$

With a uniform flow $(d h / d x=0)$ the Eq. (20) is identically equal to one. Thus, the diffusion coefficients in decelerated flow can significantly exceed similar values for the accelerated flow (in several times) that explained by a significant increase in the intensity of turbulent pulsations in decelerated flow. Therefore, decelerated flows are preferable for the intensification of diffusion process for sewage dilution in the rivers.

\section{Concentration profiles for uniform and nonuniform flow}

In the case of suspended contaminants, small size of suspended particles contributes to their involvement in the turbulent flow. For a description of their movement, we used the diffusion model of turbulent transport in one-dimensional formulation [1]

$$
\frac{\partial}{\partial y}\left(v_{T} \frac{d c}{d y}\right)+W \frac{d c}{d y}=0
$$

$c$ - volume concentration; $W$ - fall velocity.

H.Rouse studies [11] showed that for a fine mixture the eddy viscosity $v_{T}$ doesn't depend on the concentration $c$ and it is determined as well as for pure water. As a result of the integration of the Eq. (21) we find

$$
v_{T} \frac{d c}{d y}+W c=C
$$

Since the left-hand side of Eq. (22) represents a complete mass balance, passing through a unit area in the vertical direction, the constant $C$ can be assumed equal to zero

$$
v_{T} \frac{d c}{d y}+W c=0
$$

For the determination of the eddy viscosity, we use the Eq. (2). Substituting Eq. (2) into Eq. (23), we have

$$
\frac{d c}{c}=-\frac{W d \eta}{{ }_{\kappa} U_{*} \eta(1-\eta)} .
$$

After integration of Eq. (24), we obtain 


$$
\ln c=\frac{W}{\kappa U_{*}} \ln \frac{1-\eta}{\eta}+C .
$$

The integration constant $\mathrm{C}$ can be determined from the condition $\eta_{0}=0,05 c=c_{0}$. As a result, we find

$$
\ln \frac{c}{c_{0}}=\frac{W}{\kappa U_{*}}\left(\ln \frac{1-\eta}{\eta}-\ln \frac{1-\eta_{0}}{\eta_{0}}\right)
$$

Transform the Eq. (26) to the form

$$
\frac{c}{c_{0}}=\left[\frac{\eta_{0}}{1-\eta_{0}}\left(\frac{1-\eta}{\eta}\right)\right]^{\frac{\mathrm{W}}{\kappa \mathrm{U}_{*}}} .
$$

Substituting into Eq. (27) von Kármán parameter $\left(\kappa_{p}\right)$ and friction velocity $\left(U_{*}\right)$, corresponding to the conditions of uniform flow, we arrive at a well-known profile RouseVelikanov [12]

$$
\frac{c}{c_{0}}=\left[\frac{\eta_{0}}{1-\eta_{0}}\left(\frac{1-\eta}{\eta}\right)\right]^{\frac{\mathrm{w}}{\kappa_{p} \mathrm{U}_{*_{p}}}} .
$$

Consider possible changes in the concentration profiles in the case of non-uniform flow. To do this, multiply and divide the exponent in Eq. (28) on the same expression $\kappa_{p} U_{*_{p}}$ different from zero

$$
\frac{c}{c_{0}}=\left[\frac{\eta_{0}}{1-\eta_{0}}\left(\frac{1-\eta}{\eta}\right)\right]^{\frac{\mathrm{W}}{\kappa_{p} \mathrm{U}_{*_{\mathrm{p}}}} \frac{\kappa_{p} \mathrm{U}_{*_{\mathrm{p}}}}{\kappa \mathrm{U}_{*}}} .
$$

For the ratio $\kappa U_{*} / \kappa_{p} U_{*_{p}}$ previously were obtained Eq. (15) and Eq. (17). Substituting them into Eq. (29), we find for decelerated flow

$$
\frac{c}{c_{0}}=\left[\frac{\eta_{0}}{1-\eta_{0}}\left(\frac{1-\eta}{\eta}\right)\right]^{\frac{\mathrm{w}}{\kappa_{p} \mathrm{U}_{*_{\mathrm{p}}}\left(1+85,2 \frac{d h}{d x}\right)}} .
$$

The expression to accelerate the flow has the form

$$
\frac{c}{c_{0}}=\left[\frac{\eta_{0}}{1-\eta_{0}}\left(\frac{1-\eta}{\eta}\right)\right]^{\frac{\mathrm{w}}{\kappa_{p} \mathrm{U}_{\mathrm{*}_{\mathrm{p}}}\left(1-12,5 \frac{d h}{d x}\right)}} .
$$

These profiles (30) and (31) differ from the Rouse-Velikanov concentration profile (28) by amendments to the exponent that take into account the degree of non-uniformity of flow. The graph (Fig. 2) shows that the resulting profiles are characterized by a more uniform distribution of the fine mixture along of the flow depth in non-uniform flow and close to the profiles of Rouse-Velikanov [12] and A.V.Karaushev [13]. 


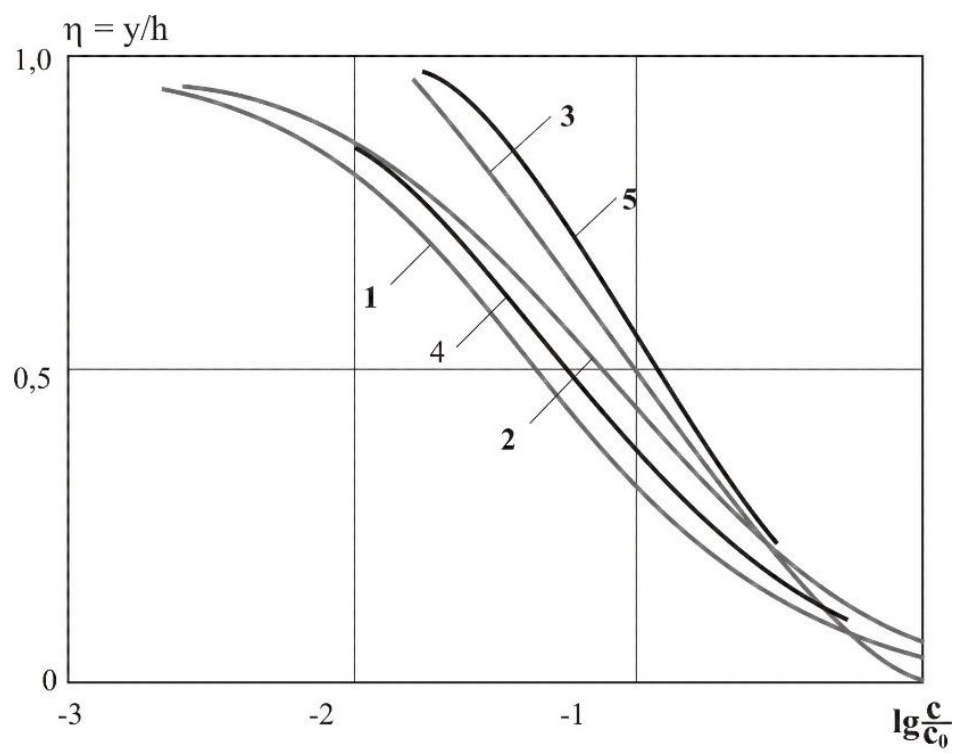

Fig. 2. Concentration profiles for unifom and nonuniform gradually varied flow:

1 - Rouse-Velikanov concentration profile, 2 - Data of V.Borovkov; 3 - data of A.Karaushev; 4 accelerated flow; 5 - decelerated flow

The average concentration of fine mixture along of the flow depth can be determined by integrating of Eq. (27), after presenting it in the form

$$
\frac{c}{c_{0}}=\left(\frac{\eta_{0}}{1-\eta_{0}}\right)^{\frac{\mathrm{W}}{\kappa \mathrm{U}_{*}}}\left(\frac{1-\eta}{\eta}\right)^{\frac{\mathrm{W}}{\kappa \mathrm{U}_{*}}} .
$$

After decomposition the variable numerator of the second factor in the binomial set, we perform integration the Eq. (32) along of the flow depth, limiting to first two terms of the expansion

$$
\frac{\bar{c}}{c_{0}}=\frac{\left(\frac{\eta_{0}}{1-\eta_{0}}\right)^{\frac{\mathrm{W}}{\kappa \mathrm{U}_{*}}}}{1-\frac{\mathrm{W}}{\kappa \mathrm{U}_{*}}}
$$

$\bar{c}$ - average concentration of suspended contaminants.

Substituting into Eq. (33) von Kármán parameter value $\left(\kappa_{p}\right)$ and friction velocity $\left(U_{* \mathrm{p}}\right)$, corresponding to the conditions of uniform flow, we have for uniform flow [1]

$$
\frac{\bar{c}}{c_{0}}=\frac{\left(\frac{\eta_{0}}{1-\eta_{0}}\right)^{\frac{\mathrm{W}}{\kappa_{p} \mathrm{U}_{*} p}}}{1-\frac{\mathrm{W}}{\kappa_{p} \mathrm{U}_{* \mathrm{p}}}} .
$$
flow

Using the previously found Eq. (15) and Eq. (17), transform Eq. (33) for decelerated 


$$
\frac{\bar{c}}{c_{0}}=\frac{\left(\frac{\eta_{0}}{1-\eta_{0}}\right)^{\frac{\mathrm{W}}{\kappa_{p} \mathrm{U}_{*_{\mathrm{p}}}\left(1+85,2 \frac{d h}{d x}\right)}}}{1-\frac{\mathrm{W}}{\kappa_{p} \mathrm{U}_{*_{\mathrm{p}}}\left(1+85,2 \frac{d h}{d x}\right)}} .
$$

The expression to accelerate the flow has the form

$$
\frac{\bar{c}}{c_{0}}=\frac{\left(\frac{\eta_{0}}{1-\eta_{0}}\right) \overline{\kappa_{p} \mathrm{U}_{*_{\mathrm{p}}}\left(1-12,5 \frac{\mathrm{W} h}{d x}\right)}}{1-\frac{\mathrm{W}}{\kappa_{p} \mathrm{U}_{*_{\mathrm{p}}}\left(1-12,5 \frac{d h}{d x}\right)}} .
$$

Curves built in accordance with (35) and (36) at $/ d h / d x /=0,005$, are represented on the graph (Fig. 3). On the same graph are plotted the experimental points obtained based on measurements on the Moscow River in the flood [1].

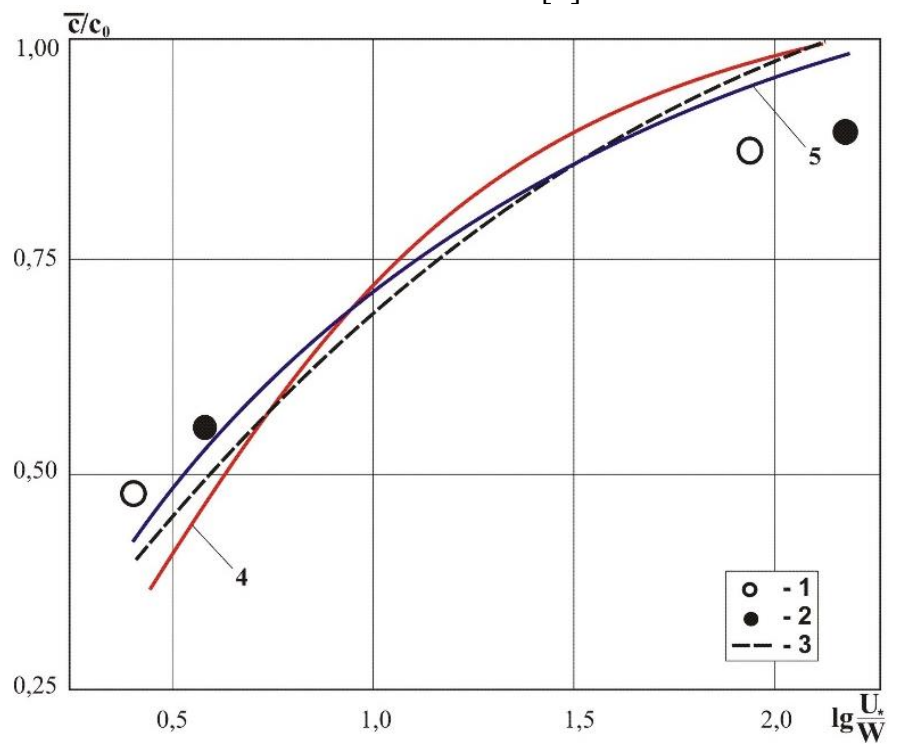

Fig. 3. The average concentration of fine mixture of the flow depth for uniform and nonuniform gradually varied flow when $\mathrm{ldh} / \mathrm{dxl}=0,005$ :

1,2 - measurements on the Moskva-river in the flood; 2 - data of V. Borovkov; 4 - data of decelerated flow; 5 - data of accelerated flow.

The graph shows that the uneven distribution of suspended contaminants along the depth of gradually varied nonuniform flow increases considerably at minimum $U_{*} / W$ values. 


\section{The deposition length for nonuniform flow}

The accumulation of fine contaminating particles on the bottom sediments affects the bed erosion and sediment transport, worsens the sanitary condition of the river [13]. According to [1] let's use the fine particles deposition scheme supposing sediments balance for a unit length section of the flow in the absence of bed erosion

$$
h d c=-c W d t
$$

$c$ - volume concentration of suspended contaminants; $t$ - time.

The solution of Eq. (20) with the initial conditions $t=0, c=c_{0}$ has the form

$$
c / c_{0}=e^{-\frac{W t}{h}} \text {. }
$$

As follows from Eq. (21), the most intense sedimentation by fine suspended particles should be observed in the areas of flow with shallow depths (coastal zones). Considering the deposition process at the section of the flow from cross-section with the initial concentration $c_{0}$ to cross-section with the residual concentration of $c=0,05 c_{0}$ we find from Eq. (38)

$$
W t / h_{c}=3,
$$

$h_{c}$ - average depth for the considered flow section.

Consequently, the time of suspension deposition

$$
t=3 h_{c} / W \text {. }
$$

Defining the time $t$ using the mean flow velocity $V$ for the flow section, we have

$$
t=L / V \text {, }
$$

$L$ - length of the deposition.

Substituting Eq. (40) into Eq. (39), we have

$$
L / h=3 V / W \text {. }
$$

Calculate the mean velocity $V$ using $U_{*}$ and $\lambda$ from the Eq. (8) for nonuniform gradually varied flow

$$
2\left(\frac{U_{*}}{V}\right)^{2}=C_{f}=\lambda\left(1-12,5 \frac{d h}{d x}\right),
$$

$d h / d x$ - average slope of the free surface for the considered flow section; $\lambda$ - hydraulic resistance coefficient for nonuniform flow.

Consequently,

$$
V=\frac{\sqrt{2} U_{*}}{\sqrt{\lambda\left(1-12.5 \frac{d h}{d x}\right)}} .
$$

Substituting Eq. (43) into Eq. (41), we find after simple transformations

$$
\frac{L}{h}=\frac{4,2}{\sqrt{\lambda\left(1-12,5 \frac{d h}{d x}\right)}} \frac{U_{*}}{W} .
$$

With a uniform flow $\mathrm{dh} / \mathrm{dx}=0$ and the Eq. (44) takes the form [3]

$$
\frac{L}{h}=\frac{4,2}{\sqrt{\lambda_{\mathrm{p}}}} \frac{U_{*}}{W}
$$

In the case of decelerated flow, we use the expression (9) for substitution into Eq. (44). After simple transformations we obtain

$$
\frac{L}{h}=\frac{4,2}{\sqrt{\lambda_{p}\left(1+8,7 \frac{d h}{d x}\right)}} \frac{U_{*}}{W} .
$$

For accelerated flow we take $\lambda=\lambda_{p}$ [4] and according to Eq. (44) the resulting relation for hydraulic resistance coefficient has the form

$$
\frac{L}{h}=\frac{4,2}{\sqrt{\lambda_{p}\left(1-12,5 \frac{d h}{d x}\right)}} \frac{U_{*}}{W} .
$$


We perform rationing for deposition lengths defined by Eq. (45) and Eq. (46) using the deposition length of equivalent uniform flow. For equal parameters $U_{*} / W$ we obtain the relationships presented below.

For decelerated flow

For accelerated flow

$$
\frac{L_{3}}{L_{\mathrm{p}}}=\frac{1}{\sqrt{\left(1+8,7 \frac{d h}{d x}\right)}} .
$$

$$
\frac{L_{y}}{L_{\mathrm{p}}}=\frac{1}{\left(1-12,5 \frac{d h}{d x}\right)} .
$$

From the analysis of Eq. (47) and Eq. (48) follows that the deposition length for the fine particles in the decelerated streams at the same value of the hydraulic resistance coefficient $\lambda \mathrm{p}$ and parameter $U_{*} / \mathrm{W}$ slightly less (within $10 \%$ ) than the corresponding value for the uniform flow under other equal conditions. For accelerated flow, the deposition length is longer than the same length for uniform flow.

\section{Conclusions}

1. The effect of flow nonuniformity on the diffusion model of wastewater dilution was investigated.

2. A method was proposed for calculating the coefficient of turbulent diffusion for open channels flows.

3. The formulas for concentration profiles and the average concentration of fine suspensions were obtained.

4. The characteristic features of sedimentation in nonuniform open flows are revealed.

\section{References}

1. V.S. Borovkov, Channel processes and dynamics of river flows in urban areas (1986)

2. K.V. Grishanin, Hydraulic resistance of natural riverbeds (1992)

3. Bennet C.O., Myers J.E., Momentum, heat and mass transfer (1966)

4. V.Yu. Lyapin, Hydraulic resistance gradually varied nonuniform and uniform flow in open channels (2004)

5. V.Yu. Lyapin, Calculations of hydraulic structures (1982)

6. F. Engelund, Energy and Momentum Equation for Non-Uniform Flow (1981)

7. I. Nikuradze, Untersuchungen Uber die Stromungen des Wassers in Konvergenten und Divergenten Kanalen (1929)

8. F. Donch, Divergence und Convergente Turbulent Stromungen mit Kleinen Offnungswinkeln (1929)

9. G.A. Gurzhienko, On a steady turbulent flow in conical diffusers with a small expansion angles (1939)

10. A.D. Altschul, Hydraulic resistance (1982)

11. H. Rouse, Fluid Mechanics for hydraulic engineers (1967)

12. M.A. Velikanov, Riverbed process (1958)

13. A.V. Karaushev, Methodical bases of an estimation and regulation of human impact on surface water quality (1987) 